\title{
Development of a Quick Reference Table for Setting Programmable Pressure Valves in Patients With Idiopathic Normal Pressure Hydrocephalus
}

\author{
Hiroji MiYAKE, Yoshinaga KAJIMOTO*, Masao TSUjI, \\ Tohru UKITA, Adam TUCKER, and Takehisa OHMURA \\ Nishinomiya Kyoritsu Neurosurgical Hospital, Nishinomiya, Hyogo; \\ ${ }^{*}$ Department of Neurosurgery, Osaka Medical College, Takatsuki, Osaka
}

\begin{abstract}
Quick and reliable setting of programmable pressure valves (PPVs) is important in the treatment of idiopathic normal pressure hydrocephalus (iNPH), especially for reducing overdrainage complications and related medical costs. A new quick reference table (QRT) was developed for improved PPV control and outcome. Shunt control can be based on the pressure environment in the sitting condition, given as hydrostatic pressure (HP) = intracranial pressure + PPV setting + intraabdominal pressure (IAP). Using this relationship, and estimating HP and IAP from the patient's height and body mass index, respectively, a QRT was designed, consisting of a matrix of the patient's height and weight. The QRT was used to make initial PPV settings in 25 patients with iNPH and the clinical outcomes were evaluated. Postoperative readjustments of the PPV were not necessary in 15 of the 25 patients. At 1 month after operation, the PPV setting was decreased once in 5 patients and increased once in 2 patients. Four of these 7 patients improved after a single readjustment. Three patients required further readjustments. At 3 months after operation, another 3 patients required a single readjustment and all improved after this readjustment. The readjustment rate was $40 \%$ and readjustment number was 0.68 times/patient. The mean PPV setting at 1 year after operation was $15.5 \pm 3.9 \mathrm{cmH}_{2} \mathrm{O}$. Use of the QRT in non-bedridden iNPH patients results in a low incidence of PPV readjustment.
\end{abstract}

Key words: idiopathic normal pressure hydrocephalus, initial pressure setting, programmable pressure valve, readjustment

\section{Introduction}

Idiopathic normal pressure hydrocephalus (iNPH) is becoming more common with the ever-growing aging population in Japan, so there is an increasing societal requirement for effective treatment of patients with iNPH. Programmable pressure valves (PPVs) have decreased the incidence of overdrainage complications, but significant risk still exists until a suitable setting of the PPV is obtained because of the low intracranial compliance in patients with iNPH. Various methods for the initial PPV adjustment have been proposed, ${ }^{1,6,8,10,12-14)}$ but no established guidelines for determining the initial pressure setting of PPVs. ${ }^{7)}$ We previously described a new concept for shunt control, which recommends setting the PPV based on the pressure environment in the upright position and the patient's physique. ${ }^{4,6)}$

Received January 15, 2008; Accepted June 17, 2008
To improve the practical availability of our concept, we have developed a quick reference table (QRT) for the pressure setting of the PPV and evaluated its clinical usefulness.

\section{Materials and Methods}

Our previous study revealed that the pressure environment of the shunted patient in the sitting position could be described by the following formula: Hydrostatic pressure (HP) = intracranial pressure (ICP) + PPV setting + intraabdominal pressure (IAP) (Fig. 1). Originally, we individually measured HP from the patient's sitting height, ICP by a telemetric ICP sensor (OSAKA telesensor; Nagano Keiki Seisakusho Co. Ltd., Tokyo), and IAP by intrabladder pressure in the vacant condition through a urethral catheter. However, the body mass index (BMI) is positively related to the IAP, ${ }^{3,9)}$ so we estimated the IAP using a graph of the relationship between 


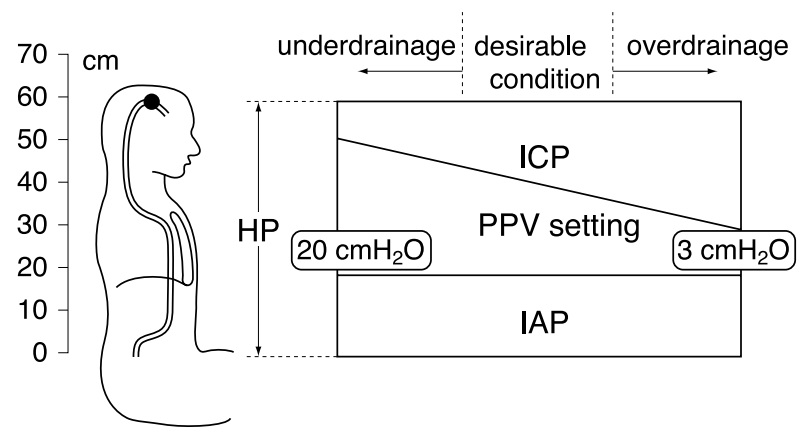

Fig. 1 Schematic illustration showing the pressure environment of a patient with shunt in the sitting position. HP: hydrostatic pressure, IAP: intraabdominal pressure, ICP: intracranial pressure, PPV: programmable pressure valve.

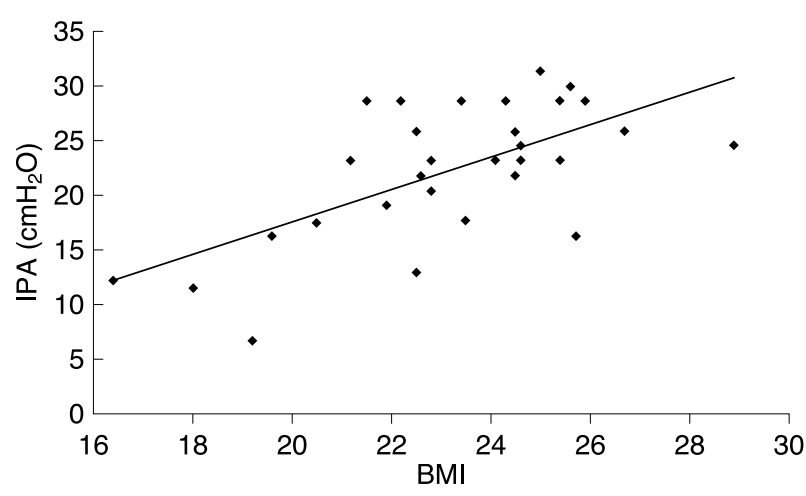

Fig. 2 Relationship between intraabdominal pressure (IAP) and body mass index (BMI) in the sitting position in 30 patients with idiopathic normal pressure hydrocephalus. $Y=1.47 X-11.73, R^{2}=0.41$.

the intrabladder pressure and the BMI, derived from data on 30 patients with iNPH at the Osaka Medical College (Fig. 2). Similarly, a relationship between sitting height and standing height was established from data on 54 elderly volunteers (all over 65 years of age, 23 men and 31 women) at the Nishinomiya Kyoritsu Neurosurgical Hospital. The sitting height and standing height showed good correlation (Fig. 3), suggesting that the sitting height can be estimated from the standing height. Our previous studies also revealed that mean ICP in the sitting position in patients with clinical improvement without postoperative complications ranged from approximately -21 to $-28 \mathrm{cmH}_{2} \mathrm{O}$ in reference to the frontal burr hole. ${ }^{4-6)}$ Based on these results, $-26 \mathrm{cmH}_{2} \mathrm{O}$ was chosen as the most desirable sitting ICP. Finally, the QRT was developed as a matrix of patient's height
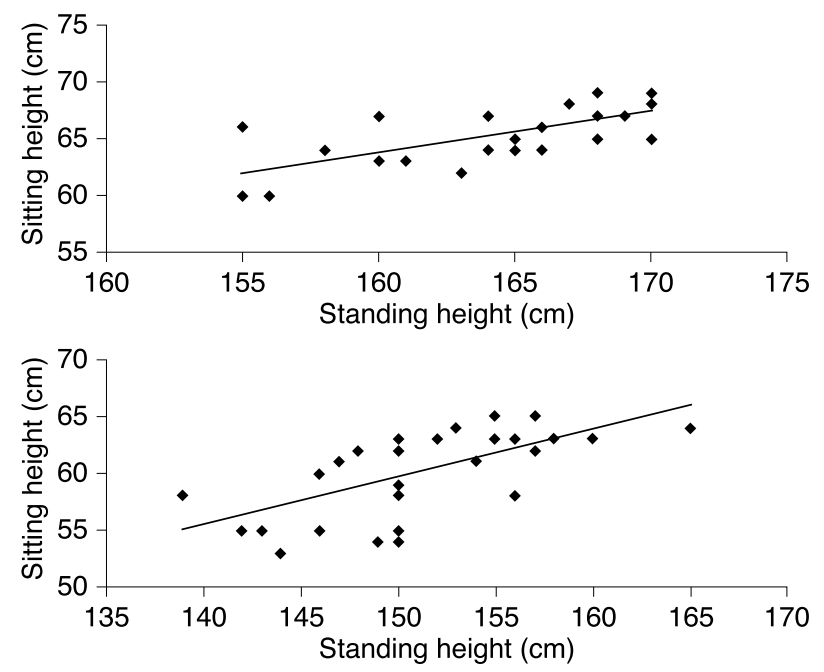

Fig. 3 Relationship between the sitting height and standing height in males (upper: $\mathrm{Y}=\mathbf{0 . 3 7 X}$ +5.15 ) and females (lower: $Y=0.42 X-$ 3.44) among 64 elderly volunteers.

and weight, as shown in Table 1 .

We used the QRT for the initial pressure setting of the PPV and investigated the number and the degree of PPV setting changes (readjustments), incidence of complications, and clinical course in 25 patients with iNPH, 16 men and 9 women (mean age 76 years), treated with ventriculoperitoneal (VP) shunting at the Nishinomiya Kyoritsu Neurosurgical Hospital for longer than 12 months between June 2003 and February 2007.

\section{Results}

All patients showed clinical improvement after the VP shunt procedure. Readjustments of the PPV setting were not necessary in 15 of the 25 patients during the entire follow-up period. The courses of the 10 patients who required readjustment are shown in Fig. 4. At 1 month after operation, 5 patients required single decrease of the PPV setting, 3 with unsatisfactory clinical improvement, 1 with progressive ventricular enlargement, and 1 with deterioration due to viral meningitis; and 2 patients required single increase of the PPV setting, 1 with low pressure headache (LPH) and 1 with subdural fluid collection (SFC). Single readjustment of the PPV resulted in clinical improvement in 4 patients, 2 with previous unsatisfactory clinical improvement and 2 with previous progressive ventricular enlargement and LPH. Three patients required a second readjustment, one for underdrainage due to excessive PPV setting increase, one for unsatisfactory clinical im- 
Table 1 Quick reference table for the initial pressure setting $\left(\mathrm{CmH}_{2} \mathrm{O}\right)$ of the programmable pressure valve (PPV) For females:

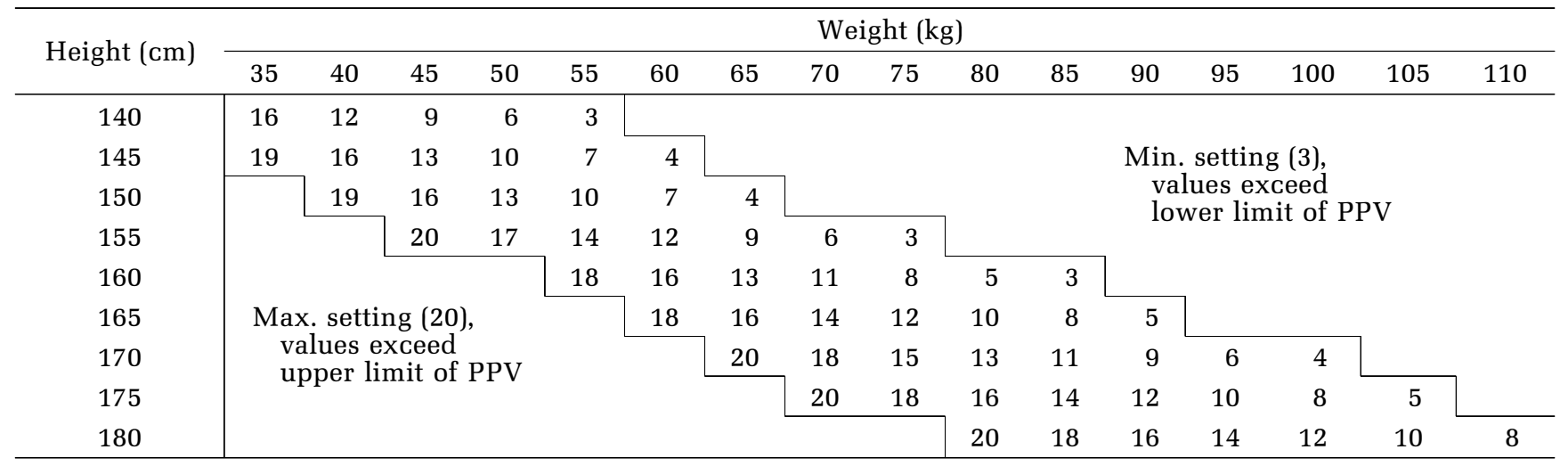

For males:

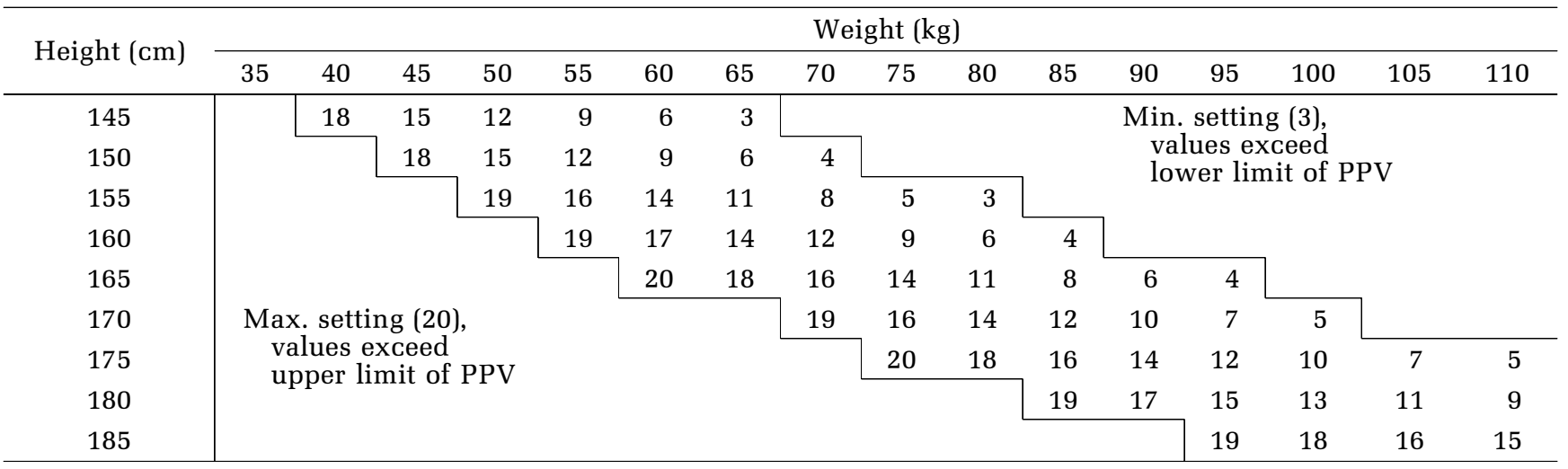

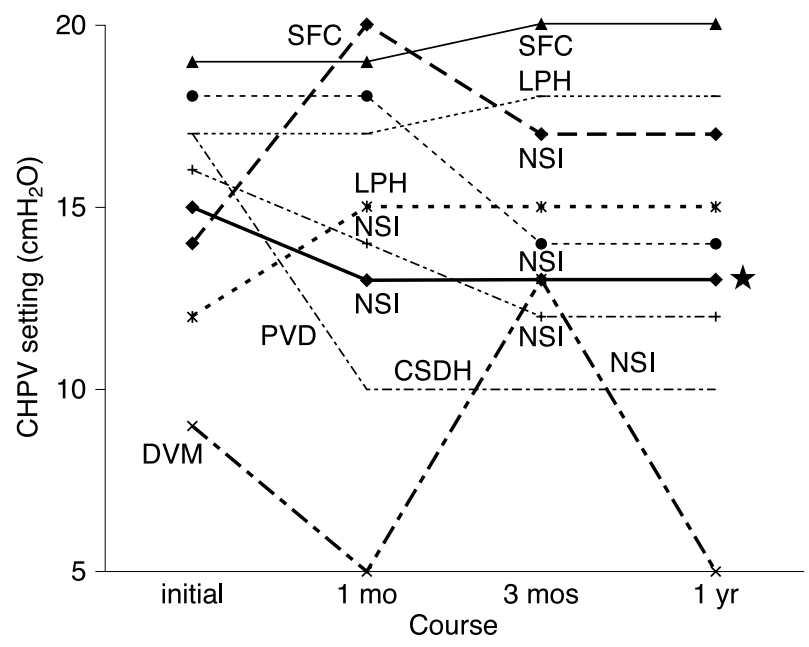

Fig. 4 Course of 10 patients who required postoperative readjustments. CHPV: Codman-Hakim programmable valve, CSDH: chronic subdural hematoma, DVM: deterioration due to viral meningitis, $L P H$ : low pressure headache, NSI: no satisfactory improvement, PVD: progressive ventricular dilation, SFC: subdural fluid collection. $\star$ Represents 2 patients. provement due to insufficient PPV setting decrease, and another for chronic subdural hematoma (CSDH) due to excessive PPV setting decrease at 1 month after operation; and all patients improved after this second readjustment. However, the patient with CSDH gradually showed underdrainage symptoms due to excessive PPV setting increase, and required a third readjustment. At 3 months after operation, another 3 patients required a single readjustment for unsatisfactory clinical improvement, SFC, and LPH. These 3 patients improved clinically after the single readjustment. No surgical intervention was necessary for the patients with CSDH and SFC in this series.

The mean setting change was $3.1 \mathrm{~cm}_{2} \mathrm{O} /$ readjustment, and the mean difference between the initial and 1-year postoperative PPV settings in patients who required readjustments was $1.2 \mathrm{cmH}_{2} \mathrm{O}$. The mean PPV setting at 1 year after operation was 15.5 $\pm 3.9 \mathrm{cmH}_{2} \mathrm{O}$.

\section{Discussion}

Rapid establishment of the optimum PPV setting can improve patient outcome as well as reduce medical costs by preventing overdrainage complications and 

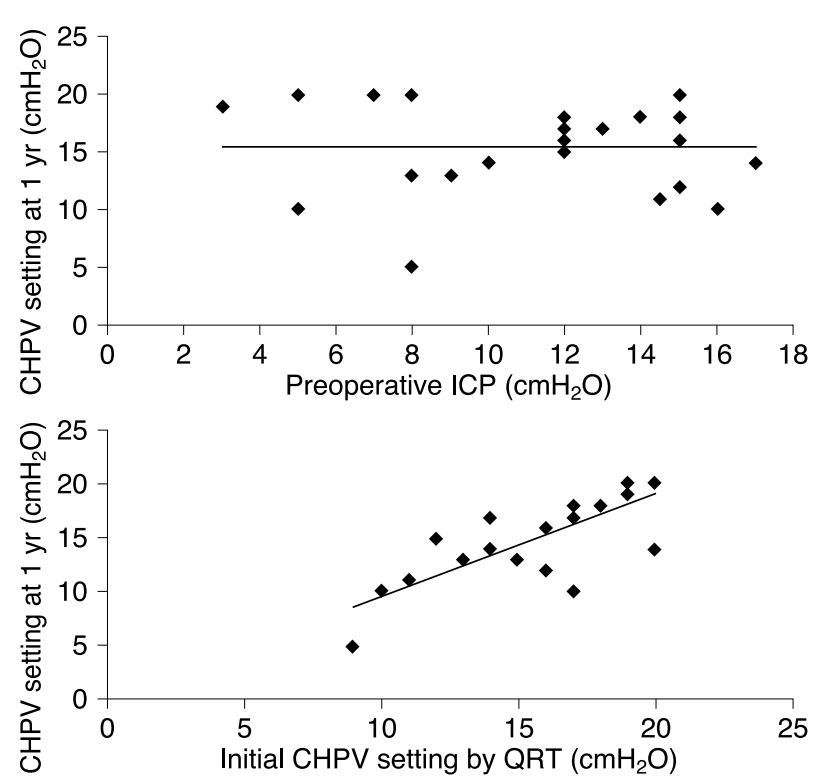

Fig. 5 Upper: Relationship between preoperative intracranial pressure (ICP) and setting of the Codman-Hakim programmable valve (CHPV) 1 year after operation. There was no correlation. Lower: Relationship between initial setting of the CHPV by the quick reference table (QRT) and the setting 1 year after operation. There was a positive correlation $\left(R^{2}=\mathbf{0 . 6 4}\right)$.

shortening hospitalization time. Starting at a low pressure setting has been recommended.13) However, low pressure valves carry greater risks of overdrainage complications ${ }^{2)}$ in patients with iNPH, and consequently are not recommended. In contrast, setting the PPV at the highest level initially was recommended, followed by decreasing the setting depending on the patient's clinical condition. ${ }^{1,8}$ Readjustment was not necessary in only 4 of 32 patients with iNPH, and the mean number of readjustments was more than 1.9 times/patient. ${ }^{8)}$ Such an approach concept may be effective for reducing overdrainage complications, but is time consuming and medicoeconomically wasteful.

A strategy for the initial PPV setting was proposed based on the patient's age, duration of the underlying disease, ventricular size, and the results of lumbar infusion test. ${ }^{14)}$ The basic approach was to set the PPV high (14-18 $\mathrm{cm} \mathrm{H}_{2} \mathrm{O}$ ) in patients with low ICP (9-13 $\left.\mathrm{cm} \mathrm{H}_{2} \mathrm{O}\right)$, and low in those with high ICP in the preoperative infusion test. A study showed 138 readjustments were performed in 147 patients with iNPH (readjustment rate of $49 \%$, with readjustment number of 0.94 times/patient). These results are better than any former method. However, our study found no correlation between the preoperative lumbar tap pressure and the PPV setting at 1 year after operation (Fig. 5 upper). Preoperative lumbar tap pressure might be important to consider for postoperative control of patients with iNPH who spend most of their daily life in bed, but such considerations are not applicable to patients who spend most of daily life sitting or standing. In the upright position, the hydrostatic pressure affects shunt flow much more than lumbar tap pressure.

Adjusting the initial PPV setting based on the preoperative cerebrospinal fluid (CSF) dynamic test was recommended with readjustments according to the results of the CSF dynamic test 2 weeks after operation. ${ }^{12)}$ Readjustment was necessary in 7 of 9 patients (readjustment rate of $78 \%$, with readjustment number of 0.9 times/patient). Furthermore, the infusion test or CSF dynamic test is invasive and difficult to perform. Another method for the initial setting of the PPV was based on patient symptoms, computed tomography (CT) findings, rate of deterioration, etc. ${ }^{10)}$ but these factors are not quantitative. Moreover, readjustment rate was $37 \%$, with readjustment number of 1.3 times/patient.

Compared to any of the above methods, our strategy for PPV settings, based on the patient's physical parameters in the sitting position, showed superior results (readjustment rate of $16 \%$, with readjustment number of 0.2 times/patient). ${ }^{6)}$ However, measurement of the patient's individual sitting height and IAP directly would be time-consuming in clinical practice. The present method of initial pressure setting using the newly developed QRT is simple, quantitative, and non-invasive with the lowest readjustment rate of $40 \%$ and readjustment number of 0.68 times/patient among all setting methods using the Codman-Hakim programmable valve, excluding our original method (Table 2).

Furthermore, initial PPV setting using the QRT showed a strong positive correlation with the PPV setting at 1 year after operation (Fig. 5 lower). The mean PPV setting at 1 year after operation was 15.5 $\pm 3.9 \mathrm{cmH}_{2} \mathrm{O}$, which was relatively higher than previously considered but very close to the value reported by Reinprecht et al.8) In addition, most readjustments were completed within 3 months after operation. Our QRT was mainly used for selecting the initial PPV setting, and subsequent readjustments were performed based on the patient's symptoms and the CT findings. However, a gain in the patient's body weight or constipation may lead to underdrainage complications due to increasing IAP in the follow-up period. ${ }^{4,6,11)}$ We believe that the QRT can be used to calculate a new PPV setting if weight change occurs, and so avoid any associated over- or 
Table 2 Comparison of various setting methods

\begin{tabular}{|c|c|c|c|c|c|c|}
\hline Author (Year) & Valve & $\begin{array}{l}\text { No. of iNPH } \\
\text { patients } \\
\text { (all NPH } \\
\text { patients) }\end{array}$ & $\begin{array}{l}\text { Readjustment } \\
\text { rate }(\%)\end{array}$ & $\begin{array}{c}\text { No. of } \\
\text { readjustments/ } \\
\text { patient }\end{array}$ & $\begin{array}{c}\text { Mean } \\
\text { setting } \\
\text { change } \\
\left(\mathrm{mmH}_{2} \mathrm{O}\right)\end{array}$ & Setting method \\
\hline $\begin{array}{l}\text { Sindou et al. } \\
(1993)^{10)}\end{array}$ & SPV & unverified (75) & 37 & 1.3 & & $\begin{array}{l}\text { symptom, } \\
\text { neuroradiological image }\end{array}$ \\
\hline $\begin{array}{l}\text { Reinprecht et al. } \\
(1995)^{8)}\end{array}$ & CHPV & $32(90)$ & 87 & $>1.9$ & & $20 \mathrm{cmH}_{2} \mathrm{O}$ \\
\hline $\begin{array}{l}\text { Miyake et al. } \\
(2000)^{6)}\end{array}$ & CHPV & $13(18)$ & 16 & 0.2 & 1.2 & $\begin{array}{l}\text { sitting condition and } \\
\text { patient's physique }\end{array}$ \\
\hline $\begin{array}{l}\text { Zemack and Romner } \\
(2002)^{14)}\end{array}$ & CHPV & $147(218)$ & 49 & 0.94 & & $\begin{array}{l}\text { infusion test, age, } \\
\text { ventricular size, } \\
\text { symptom duration }\end{array}$ \\
\hline Present series & CHPV & $25(25)$ & 40 & 0.68 & 3.1 & quick reference table \\
\hline
\end{tabular}

CHPV: Codman-Hakim programmable valve, CSF: cerebrospinal fluid, iNPH: idiopathic normal pressure hydrocephalus, SPV: Sophy programmable valve.

underdrainage complications. Therefore, the QRT can be used not only in the initial PPV setting but also in the postoperative follow-up period.

Our QRT is currently the most useful method for setting PPVs in patients with iNPH who are expected to spend postoperative daily life in the upright position, both for determining the initial PPV setting and for readjustments in accordance with changes in the patient's physique during the follow-up period. Further evaluation of the clinical usefulness of this table will be conducted in a Japanese multi-institutional randomized study of iNPH (SINPHONI: Study of Idiopathic Normal Pressure Hydrocephalus on Neurological Improvement).

\section{References}

1) Bergsneider M, Peacock WJ, Mazziotta JC, Becker DP: Beneficial effect of siphoning in treatment of adult hydrocephalus. Arch Neurol 56: 1224-1229, 1999

2) Boon AJ, Tans JT, Delwel EJ, Egeler-Peerdeman SM, Hanlo PW, Wurzer HA, Avezaat CJ, de Jong DA, Gooskens RH, Hermans J: Dutch Normal-Pressure Hydrocephalus Study: randomized comparison of low- and medium-pressure shunts. J Neurosurg 88: 490-495, 1998

3) Cobb WS, Burns JM, Kercher KW, Matthews BD, James Norton H, Todd Heniford B: Normal intraabdominal pressure in healthy adults. J Surg Res 129: 231-235, 2005

4) Kajimoto Y, Ohta T, Miyake H, Matsukawa M, Ogawa D, Nagao K, Kuroiwa T: Posture-related changes in the pressure environment of the ventriculoperitoneal shunt system. J Neurosurg 93: 614-617, 2000
5) Miyake H, Ohta T, Kajimoto Y, Matsukawa M: A new ventriculoperitoneal shunt with a telemetric intracranial pressure sensor: clinical experience in 94 patients with hydrocephalus. Neurosurgery 40: 931-935, 1997

6) Miyake H, Ohta T, Kajimoto Y, Nagao K: New concept for the pressure setting of a programmable pressure valve and measurement of in vivo shunt flow performed using a microflowmeter. Technical note. J Neurosurg 92: 181-187, 2000

7) Miyake H, Ohta T, Kajimoto Y, Ogawa D: A clinical survey of hydrocephalus and current treatment for hydrocephalus in Japan: analysis by nationwide questionnaire. Childs Nerv Syst 15: 363-368, 1999

8) Reinprecht A, Czech T, Dietrich W: Clinical experience with a new pressure-adjustable shunt valve. Acta Neurochir (Wien) 134: 119-124, 1995

9) Sanchez NC, Tenofsky PL, Dort JM, Shen LY, Helmer SD, Smith RS: What is normal intra-abdominal pressure? Am Surg 67: 243-248, 2001

10) Sindou M, Guyotat-Pelissou I, Chidiac A, Goutelle A: Transcutaneous pressure adjustable valve for the treatment of hydrocephalus and arachnoid cysts in adults. Experiences with 75 cases. Acta Neurochir (Wien) 121: 135-139, 1993

11) Sugerman HJ, DeMaria EJ, Felton WL 3rd, Nakatsuka M, Sismanis A: Increased intra-abdominal pressure and cardiac filling pressures in obesity-associated pseudotumor cerebri. Neurology 49: 507-511, 1997

12) Tsuji O, Sato K: CSF dynamics in a patient with a programmable shunt. Acta Neurochir Suppl 71: 364-367, 1998

13) Turner DA, McGeachie RE: Normal pressure hydrocephalus and dementia-evaluation and treatment. Clin Geriatr Med 4: 815-830, 1988

14) Zemack G, Romner B: Adjustable valves in normalpressure hydrocephalus: a retrospective study of 218 
patients. Neurosurgery 51: 1392-1400, 2002

Address reprint requests to: Hiroji Miyake, M.D., Director, Nishinomiya Kyoritsu Neurosurgical Hospital, 12-1 Imazuyamanaka-cho, Nishinomiya, Hyogo 6638211, Japan.

e-mail: miyake@nk-hospital.or.jp

\section{Commentary}

This experimental study by Miyake and collaborators is an important contribution to the fine tuning of the treatment of normal pressure hydrocephalus. The authors convincingly show the effect of the size and weight of the patient on the efficiency of ventriculoperitoneal drainage. The good clinical results support the notion that there is a benefit for the patients from readjustments of the valve settings based on the quick reference table. The authors comment on the effect of changes of the body weight of nonbedridden patients on the drainage function of ventriculoperitoneal shunts. Of course one may also wonder about the effect of variable activities for example with seasonal effects on the time spent taking walks or sitting in a reclining chair. The quick reference tables developed by the authors may also be considered in shunts with other causes of hydrocephalus. The authors are to be congratulated for their initiative.

Raimund FIRSCHING, M.D., L.R.C.P., M.R.C.S. Klinik für Neurochirurgie Otto-von-Guericke-Universität Magdeburg, Germany

The aging of society is proceeding worldwide, not only in Japan. Today, iNPH is established in a clinical field as a treatable dementia. CSF shunting operation is valuable for improving the symptoms of iNPH. CSF shunting operation is not difficult for a neurosurgical technique, but has critical complications such as overor underdrainage of CSF. In general, overdrainage complications are more critical than underdrainage. Accordingly, the optimum initial setting for the programmable pressure valve (PPV) is very important to prevent overdrainage complications.

The guidelines of the iNPH Association recommended initial setting for higher PPV and, if necessary, gradual adjustment. Reinprecht et al. ${ }^{2)}$ reported on the outcome in 32 cases of iNPH. They set PPV as high as $200 \mathrm{mmH}_{2} \mathrm{O}$, and obtained $75 \%$ excellent and $82 \%$ good outcomes. But 16 cases of 32 iNPH (50\%) needed readjustment of PPV. I found 536 cases of adult hydrocephalus reported from 1990 to 2002. Among these, 313 cases (58\%) of 536 cases needed readjustment of shunt valve. Miyake et al. ${ }^{1)}$ described readjustments using their personal formula (initial setting pressure $=$ hydrostatic pressure + optimum ICP(sitting) - abdominal pressure) in 3 of $18 \mathrm{iNPH}$ cases (17\%). Thus we can see the significantly decreased necessity of readjustment of postoperative setting pressure.

The new quick reference table (QRT) consists of factors such as ICP, abdominal pressure, and sitting height. The abdominal pressure can be calculated from the BMI and sitting height. The authors mentioned quick and reliable setting of PPVs is important in the treatment of iNPH. The initial setting pressure becomes more correct, the necessity for readjustment is reduced, and the medical cost of the patient should be reduced by QRT. This is a very useful article on treatment for iNPH.

\section{References}

1) Miyake H, Ohta T, Kajimoto Y, Nagao K: New concept for the pressure setting of a programmable pressure valve and measurement of in vivo shunt flow performed using a microflowmeter. Technical note. J Neurosurg 92: 181-187, 2000

2) Reinprecht A, Czeck T, Dietrich W: Clinical experience with a new pressure-adjustable shunt valve. Acta Neurochir (Wien) 134: 119-124, 1995

Takashi HaYASHI, M.D. Consultant, Division of Neurosurgery Department of Neurosciences Kawasaki Hospital Yame, Fukuoka, Japan

This study gives a quick and good method for the adjustment of programmable pressure valves in the treatment of idiopathic normal pressure hydrocephalus. I think if there are more cases included in the next clinical study, this will also prove the usefulness of this QRT, and this method which is based on the patient's physical parameters will be a very useful tool for all neurosurgeons.

Yazhuo ZHANG, M.D. Deputy Director Beijing Neurosurgical Institute Beijing, P.R.C. 